\title{
ON STARLIKE FUNCTIONS
}

J. B. TWOMEY

\section{Introduction. A function}

$$
f(z)=z+\sum_{2}^{\infty} a_{n} 2^{n}
$$

regular and univalent in $|z|<1$ is said to be starlike there if it maps $|z|<1$ onto a domain starshaped with respect to the origin. A regular function (1) is starlike in $|z|<1$ if and only if $\operatorname{Re}\left[z f^{\prime}(z) / f(z)\right]>0$ in $|z|<1$.

It was shown by the author in [2] that if $f$ is starlike then, for fixed $\arg z$,

$$
\left|\frac{z f^{\prime}(z)}{f(z)}\right| \leqq \frac{(1+o(1)) \log (4 / r)|f(z)|}{(1-r) \log (1 /(1-r))}
$$

as $|z|=r \rightarrow 1$, and further that (2) is best possible in general as far as the radial rate of growth of $z f^{\prime}(z) / f(z)$ is concerned. We remark in passing that the problem of finding the best possible lower bound for $\left|z f^{\prime}(z) / f(z)\right|$ in terms of $|f(z)|$ seems to be open.

In this note, using a different method than that in [2], we obtain a sharpened form of (2). We prove in fact the following theorem.

THEOREM. Let $f(z)=z+\sum_{2}^{\infty} a_{n} z^{n}$ be starlike in $|z|<1$. Then

$$
\left|\frac{z f^{\prime}(z)}{f(z)}\right| \leqq \frac{r \log \frac{(1+r)^{2}}{r}|f(z)|}{(1-r) \log \frac{1+r}{1-r}}+1
$$

for $|z|=r<1$. Equality holds for the starlike function $f(z)=z(1-z)^{-2}$ with $z=r$.

For univalent functions of the form (1), it is well known (see, for example, $[1$, p. 5]) that

$$
\frac{1-r}{1+r} \leqq\left|\frac{z f^{\prime}(z)}{f(z)}\right| \leqq \frac{1+z}{1-r}
$$

Received by the editors May 16, 1969. 
for $|z|=r<1$, and it may be of interest to note that the right-hand inequality of (4) is implied by (3) for starlike functions, since $|f(z)|$ $\leqq r(1-r)^{-2}$ for normalized univalent $f$ [loc. cit.].

PROOF OF THEOREM.

2. We begin by proving that

$$
\left|\frac{1+z}{1-z}\right| \leqq \frac{2 r \log \frac{1+r}{|1-z|}}{(1-r) \log \frac{1+r}{1-r}}+1
$$

for $|z|=r<1$.

The function $(x-1) / \log x$ is an increasing function of $x$ in $x>1$. Hence, for $u \geqq v>1$

$$
((u-1) / \log u) \log v+1 \geqq((v-1) / \log v) \log v+1=v .
$$

This yields (5) for the case

$$
\left|\frac{1+z}{1-z}\right|>1
$$

on putting

$$
u=(1+r) /(1-r), \quad v=\left|\frac{1+z}{1-z}\right| \quad(|z|=r<1) .
$$

For

$$
\left|\frac{1+z}{1-z}\right| \leqq 1
$$

(5) is trivial, and we have thus established (5).

3 . We now show that (3) follows easily from (5) and the representation theorem for starlike functions.

Since $\operatorname{Re}\left[z f^{\prime}(z) / f(z)\right]>0$ when $f$ is starlike, it is well known that we may write

$$
\frac{z f^{\prime}(z)}{f(z)}=\frac{1}{2 \pi} \int_{0}^{2 \pi} \frac{1+z e^{-i t}}{1-z e^{-i t}} d \mu(t)
$$

where $\mu(t)$ increases and $\mu(2 \pi)-\mu(0)=2 \pi$. From (6), by integration and the addition of $\log (1+r)^{2}$ to the resulting equation, we easily obtain

$$
\log \frac{(1+r)^{2}}{r}|f(z)|=\frac{1}{\pi} \int_{0}^{2 \pi} \log \frac{1+r}{\left|1-z e^{-i t}\right|} d \mu(t)
$$


on taking real parts.

From (6), (5) (with $z$ replaced by $z e^{-i t}$ ) and (7) it now follows that

$$
\begin{aligned}
\left|\frac{z f^{\prime}(z)}{f(z)}\right| & \leqq \frac{1}{2 \pi} \int_{0}^{2 \pi}\left|\frac{1+z e^{-i t}}{1-z e^{-i t}}\right| d \mu(t) \\
& \leqq \frac{1}{2 \pi} \int_{0}^{2 \pi}\left\{\frac{2 r \log \frac{1+r}{\left|1-z e^{-i t}\right|}}{(1-r) \log \frac{1+r}{1-r}}+1\right\} d \mu(t) \\
& =\frac{r \log \frac{(1+r)^{2}}{r}|f(z)|}{(1-r) \log \frac{1+r}{1-r}}+1 .
\end{aligned}
$$

This proves (3).

It is readily verified that we have equality in (3) for $f(z)=z(1-z)^{-2}$ with $z=r$ and we have thus completed the proof of the theorem.

I wish to thank the referee for suggesting a simplification of the proof of (5).

\section{REFERENCES}

1. W. K. Hayman, Multivalent functions, Cambridge Tracts in Math. and Math. Phys., no. 40, Cambridge Univ. Press, Cambridge, 1958. MR 21 \#7302.

2. J. B. Twomey, On the derivative of a starlike function, J. London Math. Soc. (to appear).

UNIVERSITY OF SOUTH FLORIDA 